\title{
High neopterin and IP-10 levels in cerebrospinal fluid are associated with neurotoxic tryptophan metabolites in acute central nervous system infections
}

Else Quist-Paulsen ${ }^{1,2^{*}}$ D , Pål Aukrust ${ }^{3,4,5,6,7}$, Anne-Marte Bakken Kran ${ }^{9,2,5}$, Oona Dunlop ${ }^{10}$, Vidar Ormaasen ${ }^{1}$, Birgitte Stiksrud ${ }^{1,2}$, Øivind Midttun ${ }^{8}$, Thor Ueland ${ }^{3,5,6,7}$, Per Magne Ueland ${ }^{8}$, Tom Eirik Mollnes $2,6,7,11,13,14,15$ and Anne Ma Dyrhol-Riise $1,2,6,12$

\begin{abstract}
Background: The host response to intruders in the central nervous system (CNS) may be beneficial but could also be harmful and responsible for neurologic symptoms and sequelae in CNS infections. This immune response induces the activation of the kynurenine pathway (KP) with the production of neuroactive metabolites. Herein, we explored cytokine and KP responses in cerebrospinal fluid (CSF) and serum in patients with encephalitis, aseptic, and bacterial meningitis.

Methods: Cytokines were measured in CSF and serum by multiplex assay in adult patients with encephalitis of infectious, autoimmune or unknown etiology $(n=10)$, aseptic meningitis (ASM, $n=25)$, acute bacterial meningitis (ABM, $n=6)$, and disease control patients with similar symptoms but without pleocytosis in CSF $(n=42)$. Liquid chromatography-tandem mass spectrometry (LC-MS/ MS) was used to measure KP metabolites in CSF and serum.

Results: A characteristic pattern of increasing cytokine levels and KP metabolites was found in CSF from encephalitis to ASM, with the highest levels in ABM. In ASM and ABM, most inflammatory mediators, including IL-6, IL-8, and IFNinducible protein-10 (IP-10), showed markedly elevated levels in CSF compared with serum, indicating production within the CNS. In contrast to most mediators, the highest level of IP-10 was found in the ASM group, suggesting a potential role for IP-10 in aseptic/viral meningitis. Neopterin and IP-10 were associated with marked changes in KP metabolites in CSF with increasing kynurenine/tryptophan ratio reflecting indoleamine 2,3-dioxygenase activity. Neopterin, a marker of IFN- $\gamma$ activity, was associated with an unfavorable balance between neuroprotective and neurotoxic tryptophan metabolites.

Conclusion: We show that parenchymal and meningeal inflammations in CNS share a characteristic cytokine profile with a general immune response in the CSF with limited influence from the systemic circulation. IFN- $\gamma$ activity, assessed by neopterin and IP-10 levels, may play a role in the activation of the KP pathway in these patients, potentially mediating neurotoxic effects.
\end{abstract}

Keywords: Encephalitis, Aseptic meningitis, Bacterial meningitis, Cytokines, Chemokines, Kynurenine tryptophan pathway, Indoleamine 2,3-dioxygenase, Neopterin

\footnotetext{
* Correspondence: e.q.paulsen@studmed.uio.no

'Department of Infectious Diseases, Oslo University Hospital, Ullevaal

Hospital, P. O. Box 4956 Nydalen, N-0450 Oslo, Norway

${ }^{2}$ Institute of Clinical Medicine, University of Oslo, Oslo, Norway

Full list of author information is available at the end of the article
}

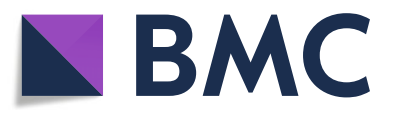

(c) The Author(s). 2018 Open Access This article is distributed under the terms of the Creative Commons Attribution 4.0 International License (http://creativecommons.org/licenses/by/4.0/), which permits unrestricted use, distribution, and reproduction in any medium, provided you give appropriate credit to the original author(s) and the source, provide a link to the Creative Commons license, and indicate if changes were made. The Creative Commons Public Domain Dedication waiver (http://creativecommons.org/publicdomain/zero/1.0/) applies to the data made available in this article, unless otherwise stated. 


\section{Background}

The host inflammatory response to intruders to the central nerve system (CNS) plays an important role for neuronal injury in encephalitis and meningitis. The cytokine profiles of aseptic meningitis (ASM) and acute bacterial meningitis (ABM) have been investigated in several studies, in general showing increased levels of inflammatory mediators [1-8]. However, for encephalitis, inflammatory responses have mainly been evaluated for patients with herpes simplex virus (HSV) infection [9-13]. Thus, comparison of cytokine levels in encephalitis, ASM and ABM and control patients are scarce. Moreover, most studies have focused on a limited number of inflammatory markers, and few studies have examined parallel samples of serum and cerebrospinal fluid (CSF) from the same patients.

It is known that the inflammation activates the kynurenine pathway (KP) resulting in the depletion of tryptophan (TRP) and formation of metabolites with potential neurotoxic (e.g., quinolinic acid [QA], 3-hydroxykynurenine [3-HK]) and neuroprotective (e.g., kynurenic acid [KYNA]) effects (Fig. 1) [14, 15]. The activation of the KP seems to also have immune modulating effects, resulting in inhibition of $\mathrm{T}_{\mathrm{H}} 1$ cells, activation of regulatory $\mathrm{T}$ cells $\left(\mathrm{T}_{\text {regs }}\right)$ and inhibition of natural killer (NK) cells [16-19]. In the CNS, the rate-limiting enzyme for TRP catabolism is indoleamine 2,3-dioxygenase (IDO) which is upregulated by inflammatory cytokines, mainly by interferon gamma (IFN- $\gamma$ ) [20], linking $\mathrm{T}$ cell activation to the regulation of the KP.

Altered cytokine levels and associated imbalance of neurotoxic and neuroprotective metabolites in the KP have been suggested to contribute to the pathogenesis of several chronic conditions in the CNS, such as schizophrenia [21, 22], bipolar disorders [23, 24], Parkinson's disease [25], Alzheimer's [26] and Huntington's disease [27], and AIDS-related dementia [28, 29], as well as in traumatic brain injury [30]. However, there is limited data on the role of KP in acute infections like encephalitis and meningitis, although increased levels of KYNA have been shown in patients with HSV encephalitis, Lyme borreliosis, tick-borne encephalitis, and bacterial meningitis [4, 31-33]. Moreover, altered tryptophan metabolism has been linked to disease severity in tuberculous meningitis [34].

The aim of the present study was to elucidate the inflammatory network and KP metabolites in ASM and $\mathrm{ABM}$, characterized by meningeal inflammation, and in encephalitis, characterized by brain parenchymal inflammation. Parallel samples of serum and CSF were examined in patient groups and control patients, i.e., patients with similar symptoms but without CSF pleocytosis or other signs of CNS infection.

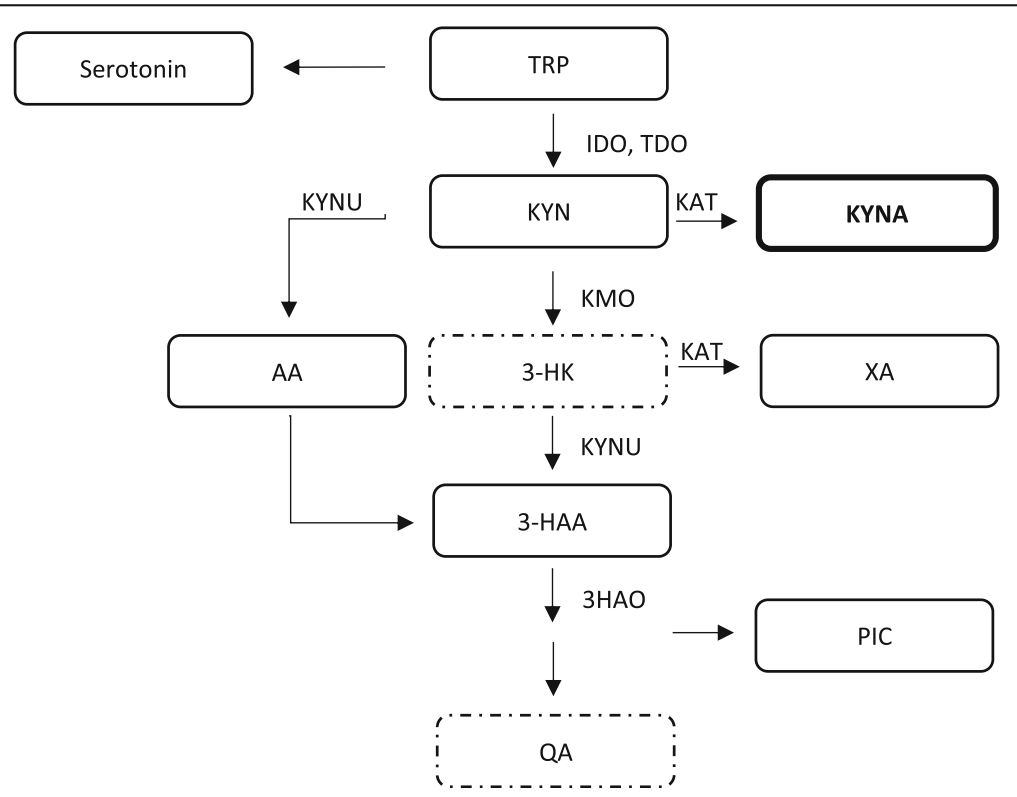

Fig. 1 Schematic presentation of the KP pathway. IDO is the main enzyme responsible for the TRP catabolism in CNS. KYN is further degraded into the neuroprotective NMDAr antagonist KYNA by KAT, or by KMO and KYNU into the neurotoxic metabolites of 3-HK and QA. QA is an agonist of the NMDA receptor. Abbreviations: AA, anthranilic acid; 3-HAA, 3-hydroxyanthranilic acid; HAO, 3-hydroxyanthranilic acid oxidase; 3-HK, 3-hydroxykynurenine; IDO, indoleamine-2,3-dioxygenase; KAT, kynurenine aminotransferase; KMO, kynurenine 3-monooxygenase; KYN, kynurenine; KYNA, kynurenic acid; KYNU, kynureninase; QA, quinolinic acid; PIC, picolinic acid; TRP, tryptophan; XA, xanthurenic acid. Bold box indicates neuroprotective metabolite, dashed boxes indicate neurotoxic metabolites in the KP pathway 


\section{Methods}

\section{Study participants and study design}

This cross-sectional study was performed at the Oslo University Hospital (OUS), Ullevaal, a regional hospital for 2.7 million people. Patients were eligible for inclusion if they had (1) onset of symptoms of CNS infection within less than 7 days and (2) clinical indication for a diagnostic lumbar puncture (LP). Patients were included between January 2014 and December 2015. CSF leukocyte counts $\geq 5 \times 10^{6} / \mathrm{L}$ was found in 68 patients. Of these, $23 \mathrm{did}$ not fulfill the case definition of encephalitis of viral, autoimmune or unknown cause, aseptic meningitis (ASM), or bacterial meningitis (ABM) (Additional file 1: Table S1). In four patients, the time from CSF sampling until centrifugation was $>10 \mathrm{~h}$, rendering a total of 41 patients with CNS infection (Additional file 2: Figure S1). The control group consisted of age- and gender-matched patients with similar symptoms, but without signs of CNS inflammation, i.e., no pleocytosis and no microbiological agent detected in their CSF $(n=42)$. In the control group, patients with delirium, chronic or acute psychiatric disease, Parkinson's disease, Huntington's disease, CNS malignancy, dementia, epilepsy or seizures, cerebral vascular disease, transient global amnesia, and septicemia were not included. For detailed case definitions, see Additional file 1: Table S1. Flowchart of the study population and the overview of analyses performed for the various groups are shown in Additional file 2: Figure S1.

All patients, or their next of kin when the patient was not able to consent, gave written informed consent to participate in the study. The study was approved by The Regional Committees for Medical and Health Research Ethics (REC South East, reference number 2011/2578) and the ethical council of the hospital.

\section{Microbiological diagnostics}

For all included patients, CSF leukocyte counts (CSF WBC), CSF protein, and CSF glucose were measured. Bacterial culture of CSF and analyses for identification of causative agent were performed in all individuals by a panel of specific PCR for common neurotropic virus and bacteria.

\section{Sampling of CSF and serum}

CSF samples were collected in endotoxin-free polypropylene tubes and stored at $4{ }^{\circ} \mathrm{C}$ until centrifugation at $2000 \times g$ for $10 \mathrm{~min}$. Serum samples were collected in endotoxin-free tubes without any additives, allowed to clot at room temperature, and centrifuged at $3000 \times g$ for $10 \mathrm{~min}$. Supernatants from CSF and serum were centrifuged within $10 \mathrm{~h}$ after collection and immediately frozen in triplicates of approximately $700 \mu \mathrm{L}$ each at $-80^{\circ}$ C. All analyses were performed on previously unthawed samples. For six patients, no serum was available.
Multiplex analyses of soluble markers in CSF and serum A multiplex cytokine assay (Bio-Plex Pro Human Cytokine 27-plex Panel; Bio-Rad laboratories Inc. Hercules, CA) was used to measure the concentrations of 27 different cytokines: tumor necrosis factor (TNF), IFN- $\gamma$, interleukin (IL)-1 $\beta$, IL-1 receptor antagonist (IL-1Ra), IL-2, IL-4, IL-5, IL-6, IL-7, IL-8/CXCL8, IL-9, IL-10, IL-12(p70), IL-13, IL-15, IL-17A, monocyte chemoattractant protein (MCP)-1/CCL2, IFN-inducible protein-10 (IP-10)/ CXCL10, eotaxin/CCL11, macrophage inflammatory protein- $1 \alpha$ and $-1 \beta$ (MIP- $1 \alpha / C C L 3$, MIP- $1 \beta / C C L 4)$, regulated on activation, normal $\mathrm{T}$ cell expressed and secreted (RANTES/CCL5), granulocyte-colony stimulating factor (G-CSF), granulocyte-macrophage CSF (GM-CSF), basic fibroblast growth factor (FGF), platelet-derived growth factor (PDGF), and vascular endothelial growth factor (VEGF). The assay was performed using the instructions of the manufacturer. CSF samples were tested undiluted. Only cytokines with less than 20\% missing values were included in further analyses. Undetectable levels were assigned the lowest detectable level (LDL) measured in the cohort for the respective marker. In the CSF samples, IFN- $\gamma$, IL-5, PDGF, Basic FGF, and RANTES, and for serum, IL-2, IL-5, IL-7, IL-15, G-CSF, GM-CSF, and FGF were excluded from further analyses based on the criteria stated above.

\section{Mass spectrometry analyses of tryptophan metabolites in CSF and serum}

Tryptophan and kynurenine metabolites were measured only in the patients with encephalitis, ASM with confirmed viral etiology (viral meningitis, VM), ABM, and controls (Additional file 2: Figure S1). Concentrations of TRP, kynurenine [KYN], anthranilic acid [AA], KYNA, 3-HK, 3-hydroxyanthranilic acid [3-HAA], xanthurenic acid [XA], QA, picolinic acid [PIC], and neopterin were analyzed in CSF and serum by liquid chromatography-tandem mass spectrometry (LC-MS/ MS) by Bevital AS [35, 36]. For TRP, nine patients had levels in CSF below the lower limit of detection (LOD). As this may represent a finding rather than a limitation by the analysis, these were set equal to the LOD $(0.4 \mu \mathrm{M})$ in the statistical analyses and in the calculation of the KYN/TRP ratio (KYN (nmol)/TRP $(\mu \mathrm{mol})$ ) as an index of IDO activity. XA was not detected in CSF for 46 of the 50 patients and was not included in the analysis.

\section{Statistical methods}

Continuous data are presented as median (IQR, interquartile range). Due to lack of normal distribution, analysis of variance (ANOVA) with the Kruskal-Wallis test for multiple groups was used. If the Kruskal-Wallis test revealed significant differences, the Mann-Whitney $U$ 
test was used to compare pairs of groups. To limit type II statistical errors, no correction for multiple comparisons was made in this explorative study. $P$ values $<0.05$ were considered statistically significant. Categorical variables are expressed as counts (percentages) and analyzed by Pearson's chi-square test. Correlations were analyzed using Spearman's rank correlation coefficient. All data analyses were performed in SPSS version 24 (IBM Corp. Armonk, NY, USA) and graphs generated by GraphPad Prism 7 (GraphPad, San Diego, USA).

\section{Results}

\section{Study participant characteristics}

Ten patients had encephalitis of viral, autoimmune, or unknown etiology according to the case definition (Additional file 1: Table S1), 25 patients were diagnosed with ASM, six patients with ABM, and 42 were control patients. Characteristics of the study group are presented in Table 1 . There were no significant differences in gender or age between the patient groups. In the CNS infection group, four patients reported a history of depression, but only two of these received antidepressant drugs. The etiology of encephalitis was known for four patients (40\%), three viral (adenovirus, HSV1, varicella-zoster virus [VZV]) and one N-methyl-D-aspartate receptor [NMDAr] encephalitis. Of the 25 patients with ASM, eight were diagnosed with enterovirus in CSF, six patients suffered from HSV2 meningitis, one patient seroconverted and had positive IgM in CSF for Toscana virus, and for one patient, intrathecal antibody production of $\operatorname{IgG}$ against Borrelia burgdorferi was detected. For patients with ABM, Streptococcus pneumoniae $(n=2)$, Staphylococcus aureus $(n=2)$, Neisseria meningitidis $(n=1)$, and Haemophilus influenzae $(n=1)$ were detected in CSF by growth and/or PCR, and for all

Table 1 Patient characteristics and clinical presentation

\begin{tabular}{|c|c|c|c|c|c|}
\hline Parameter & Encephalitis $(n=10)$ & $\operatorname{ASM}(n=25)$ & $\mathrm{ABM}(n=6)$ & Controls $(n=42)$ & $p$ value $^{\mathrm{a}}$ \\
\hline Gender, males (\%) & $4(40)$ & $10(40)$ & $4(67)$ & $13(31)$ & 0.385 \\
\hline Age, years & $43.5(30,72)$ & $35(28,48)$ & $52(41,68)$ & $31(22,41)$ & 0.054 \\
\hline Hospital stay, days & $19(11,42)^{b}$ & $3(1.5,5.5)^{c}$ & $19(14,33)^{b, d}$ & $2.0(1.0,4.0)$ & $<0.001$ \\
\hline \multicolumn{6}{|l|}{ Comorbidity (\%) } \\
\hline Immunodeficiency ${ }^{\mathrm{e}}$ & $2(30)^{b}$ & $1(4)^{c}$ & $1(17)^{b}$ & - & 0.029 \\
\hline Psychiatric disorder & $2(20)^{b}$ & $2(8)$ & - & - & 0.046 \\
\hline Etiology known (\%) & $4 / 10(40)$ & $16 / 25(64)$ & $6 / 6(100)$ & - & $<0.001$ \\
\hline Headache (\%) & $7 / 10(70)^{b}$ & $24 / 25(96)^{c}$ & $3 / 4(75)$ & 39/41 (95) & 0.037 \\
\hline Neck stiffness $(\%)^{9}$ & $2 / 10(20)$ & $12 / 25(48)$ & $3 / 5(60)$ & 12/42 (29) & 0.175 \\
\hline Objective fever $(\%)^{h}$ & $7 / 10(70)$ & $17 / 25(68)$ & $5 / 6(83)$ & 20/42 (48) & 0.168 \\
\hline Focal neurology (\%) & $5 / 9(56)^{b}$ & $2 / 19(10)^{c}$ & $1 / 6(17)$ & $1 / 24(4)$ & 0.003 \\
\hline GCS $\leq 14(\%)$ & $10 / 10(100)^{b}$ & $2 / 25(8)^{c}$ & $5 / 6(83)^{b, d}$ & $6 / 42(14)$ & $<0.001$ \\
\hline $\operatorname{CSF} \mathrm{WBC}\left(\times 10^{6} / \mathrm{L}\right)$ & $25(9.5,92)^{b}$ & $179(26,271)^{b, c}$ & $212(91,1434)^{b, c}$ & $1.0(1.0,2.0)$ & $<0.001$ \\
\hline CSF protein (g/L) & $0.57(0.4,0.9)^{b}$ & $0.59(0.4,0.8)^{b}$ & $2.2(0.8,5.9)^{b, c, d}$ & $0.26(0.2,0.3)$ & $<0.001$ \\
\hline CSF glucose $(\mathrm{mmol} / \mathrm{L})$ & $3.6(3.3,4.4)$ & $3.5(3.0,3.7)$ & $3.6(0.4,6.3)$ & $3.5(3.2,3.7)$ & 0.341 \\
\hline Glucose ratio & $0.6(0.5,0.7)$ & $0.6(0.5,0.6)^{b}$ & $0.4(0.1,0.6)^{b}$ & $0.6(0.6,0.7)$ & 0.009 \\
\hline Albumin ratio & $8.4(6.5,14)^{b}$ & $9.4(6.1,13)^{b}$ & $79(36,137)^{b, c, d}$ & $4.0(2.6,5.0)$ & $<0.001$ \\
\hline Blood WBC $\left(\times 10^{9} / \mathrm{L}\right)$ & $8.6(7.4,11)$ & $8.2(6.1,11)$ & $14(8.5,18)$ & $10(6.7,12)$ & 0.173 \\
\hline CRP, serum (mg/L) & $6(0.9,86)$ & $3.5(1.3,12)^{b}$ & $169(96,446)^{b, c, d}$ & $16(2.1,77)$ & 0.001 \\
\hline
\end{tabular}

CSF WBC white blood cell count in CSF, glucose ratio CSF glucose/serum glucose, albumin ratio CSF albumin/serum albumin. Significant $p$-values are marked in bold.

Data shown are median (IQR) or numbers $/ n(\%)$

${ }^{a} p$ values for one way analysis of variance (Kruskal-Wallis)

${ }^{\mathrm{b}} p<0.05$ for analysis with Mann-Whitney $U$ test (MWU) in comparison with the control group

$c_{p}<0.05$ for analysis with MWU in comparison with encephalitis

${ }^{d} p<0.05$ for analysis with MWU in comparison with ASM

'Under treatment for or treated for cancer within the last year (including hematological malignancies), HIV infection or diabetes mellitus type 2 (DM2), or using immunosuppressive or immune modulating drugs

${ }^{f}$ Causing agents for encephalitis were 3 viral (adenovirus, HSV1, VZV) and 1 NMDAr encephalitis. ABM; Streptococcus pneumonia $(n=2)$, Staphylococcus aureus $(n=$ 2), Neisseria meningitides $(n=1)$, and Haemophilus influenzae $(n=1)$. ASM; HSV2 $(n=6)$, enterovirus $(n=8)$, Toscana virus $(n=1)$, and 1 patient had intrathecal antibody production of IgG against Borrelia burgdorferi

${ }^{9}$ Neck stiffness was assessed by a physician before LP

$h^{h}$ Fever was defined as either $\geq 38^{\circ} \mathrm{C}$ upon admission or within $24 \mathrm{~h}$ after admission, or measured to $\geq 38{ }^{\circ} \mathrm{C}$ by the patient prior to admission 
these patients, the causative bacteria was also detected in blood culture. Patients with encephalitis presented with less headache and more focal neurology compared to the other groups, and impairment of consciousness was observed in significantly fewer patients with ASM and in the control group. Importantly, the majority of the control group had fever, headache, and neck stiffness, similar to most of the patients with CNS infection.

\section{Cytokine profiles in CSF and serum}

Cytokines analyzed in parallel in CSF and serum showed distinct patterns for the different patient groups. Overall, the highest levels of CSF cytokines were found in patients with $A B M$, including the prototypical inflammatory cytokines TNF, IL-1 $\beta$ and IL-6, inflammatory chemokines (e.g., IL-8, MCP-1, MIP- $1 \alpha$ and MIP-1 $\beta)$, cytokines with potent effect on $T$ cell function (e.g., IP-10, IL-7, IL-9 and IL-15), and growth factors (e.g., VEGF and G-CSF) (Fig. 2, Additional file 3: Table S2). The typical cytokine pattern in CSF was an increase from disease controls without CNS infection to patients with encephalitis and ASM with the highest levels in those with ABM. In contrast to this pattern, the CXC chemokine IP-10 showed the highest median level in the ASM group (Fig. 2). Although lower than in patients with $A B M$ and ASM, patients with encephalitis had higher levels for most cytokines in CSF compared to the control group, with no significant difference in the levels between encephalitis cases with or without known etiology (data not shown).

The encephalitis group and the control group generally showed lower cytokine levels in CSF than in serum, with exceptions for IL-6 and IL-8 in the encephalitis group and MCP-1 and IP-10 for both groups (Figs. 2 and 3, Additional file 3: Table S2). In contrast, the groups with meningeal involvement and in particular the ABM group displayed markedly higher CSF levels than serum levels for most cytokines. For some of the cytokines, the CSF levels were more than tenfold higher than in serum (e.g., IL-6, IL-8, IP-10). In general, serum levels did not display the striking and significant differences between the patient groups and controls as seen in CSF (Fig. 3). In fact, although all patients in the ABM group had a positive blood culture, only TNF, IL-6, IL-8, IL-1Ra, and MIP- $1 \alpha$ demonstrated higher serum levels than in the control group.

When we analyzed the CNS infections all together (encephalitis, ASM, and ABM), a significant correlation between serum and CSF levels were found only for TNF (Rho 0.4, $p=0.03$ ), IL-1Ra (Rho 0.4, $p=$ 0.03 ), IL-6 (Rho 0.5, $p=0.004$ ), and MCP-1 (Rho 0.3, $p=0.04)$ suggesting intrathecal production of most of the examined mediators (Additional file 4: Table S3). Finally, except for MCP-1, all cytokines in CSF correlated positively with the CSF leukocyte counts, and all, except for MCP-1 and IP-10, correlated with CSF/ serum albumin ratio. Collectively, these data underscore the limited information obtained from a serum sample in contrast to that obtained from CSF when examining the inflammatory network in infectious CNS diseases.

\section{Profile of tryptophan metabolites and IDO activity in CNS infections}

In order to relate the cytokine profile to tryptophan metabolism (Fig. 1), metabolites of the KP in the CSF were examined in patients with encephalitis $(n=10)$, those in the ASM group with verified viral cause (VM, $n$ $=12), \mathrm{ABM}(n=6)$, and controls $(n=22)$. The median levels of most KP metabolites were higher in patients with encephalitis, VM, and ABM compared with the controls, with the highest median levels observed in the ABM group (Fig. 4, Additional file 5: Table S4). The only exception was TRP, which was lowest in the VM group (Fig. 4).

The KYN/TRP ratio was higher in all CNS infections compared to the controls $(p=0.009)$, indicating an increased conversion of TRP (Fig. 4). Moreover, to evaluate the relationship between putatively neuroprotective (KYNA) and neurotoxic (3-HK and QA) KP metabolites, we calculated the $\mathrm{KYNA} /(3-\mathrm{HK}+\mathrm{QA})$ ratio showing decreased levels for patients with encephalitis indicating a net neurotoxic effect of TRP metabolites in these patients (Fig. 4).

Most KP metabolites were present at higher concentrations in serum (Additional file 5: Table S4, Additional file 6: Figure S2) than in CSF (Fig. 2), but with less significant differences between the groups. For the total group with CNS infection (encephalitis, VM and ABM), CSF levels correlated with serum levels for 3-HK (Rho 0.6, $p<0.001$ ), QA $(0.4, p=0.04)$, and PIC $(0.7, p<0.001)$, for all other KP metabolites, there was no significant correlation between CSF and serum levels. Furthermore, all CSF/serum ratios for KP metabolites, except for TRP, KYNA, and PIC ratio, were positively correlated with CSF WBC count, while only the ratio of PIC was correlated with CSF albumin/serum ratio (Additional file 7: Table S5).

\section{Markers of IFN- $\gamma$ activation correlate with activation of the KP}

IFN- $\gamma$ is known to have a major influence on the KP $[37,38]$. In the present study, IFN- $\gamma$ levels in CSF were below detection level in $58 \%$ of the cases. However, neopterin, being a reliable and stable marker of IFN- $\gamma$ activity [39], was markedly elevated in CSF in all groups of patients with neuroinflammation 


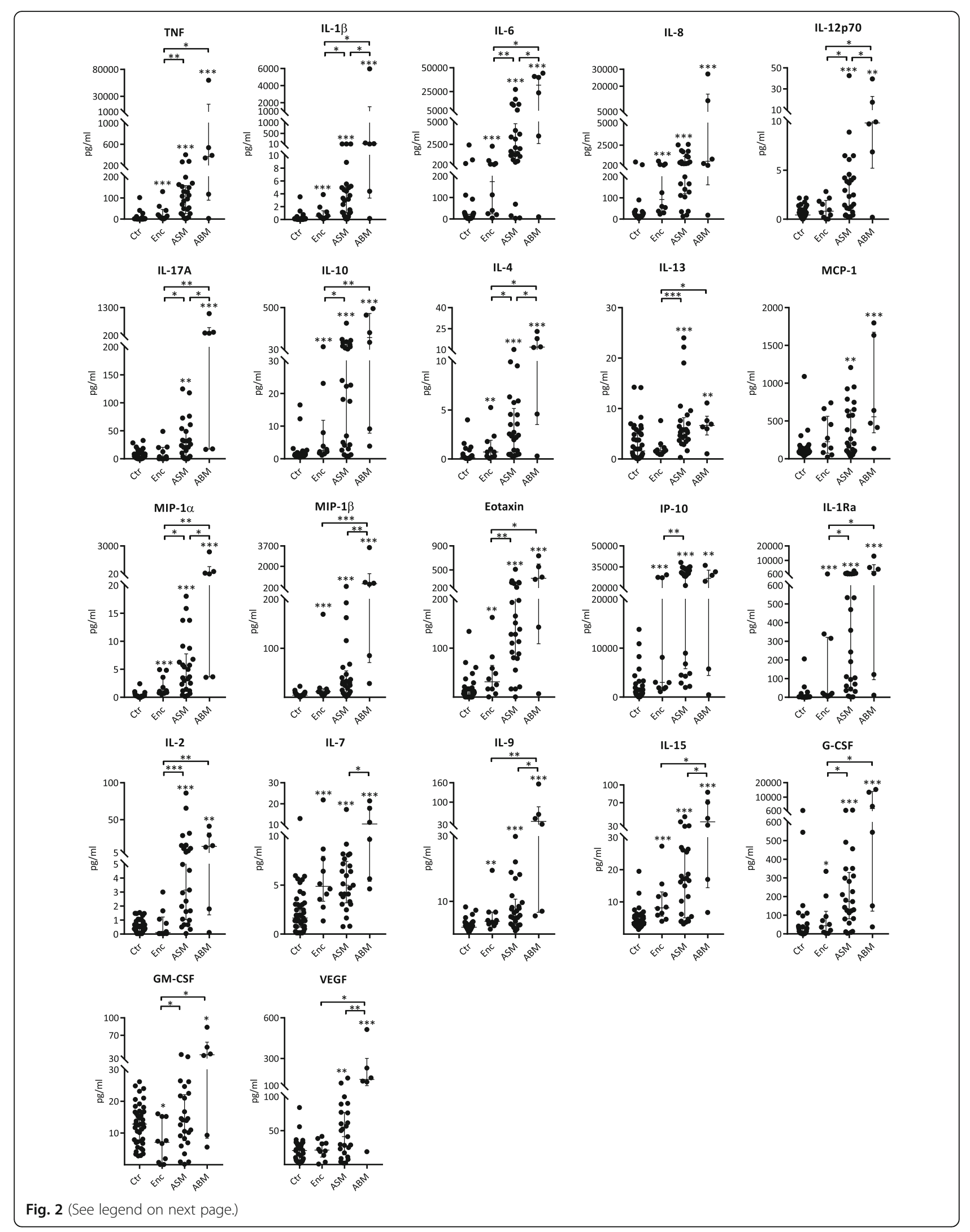


(See figure on previous page.)

Fig. 2 Cytokines in CSF in patients with encephalitis (Enc, $n=10)$, aseptic meningitis (ASM, $n=25)$, bacterial meningitis (ABM, $n=6$ ) in comparison with controls $(C t r, n=42)$. Data shown are medians with IQR and all were significant by the Kruskal-Wallis test. Comparisons of two groups were analyzed by using Mann-Whitney $U$ test. Asterisks above patient groups indicate significant difference vs controls, asterisks above horizontal lines indicate significant differences between individual groups (Mann-Whitney $U$ test): ${ }^{*} p<0.05$, ${ }^{* *} p<0.01$, and ${ }^{* * *} p<0.001$. Values below the detection limit were set to the lowest detectable level for that analyte

compared with controls (Fig. 4). Notably, when all CNS infections were analyzed together, CSF level of neopterin was strongly positively correlated with IDO (measured as the KYN/TRP ratio) and inversely correlated with the $\mathrm{KYNA} /(3-\mathrm{HK}+\mathrm{QA})$ ratio (Fig. 5). This suggests an association between high IFN- $\gamma$ activity and net neurotoxic effects of KP metabolites. A positive correlation was also seen between IDO and IP-10, another IFN- related cytokine, but no significant correlation was found between IP-10 and KYNA/(3-HK + QA) ratio (Fig. 5).

\section{Association of cytokines and metabolites in the KP and clinical variables}

Abnormal regulation of the KP metabolites has been related to depression $[40,41]$. However, excluding the two patients in the encephalitis group who reported existing or recent depression from the correlation analyses did not influence the findings (data not shown). Although the Glasgow Coma Scale (GCS) is a crude tool, it reflects the severity of critically ill patients. Among patients with aseptic meningitis, all but two had a GCS at 15; thus, only patients with encephalitis and bacterial meningitis were included in correlation analyses with GCS. We did not find any significant correlations between cytokine levels or KP metabolites in CSF and GCS in these patients (data not shown). However, the number of patients was too low to make any firm conclusion.

\section{Discussion}

In the present study, we demonstrate a gradual increase in levels of a wide range of cytokines, including chemokines, interleukins, interferons, and growth factors from encephalitis to ASM and finally to ABM as compared with controls, reflecting the level of CSF inflammation seen in patients with these CNS infections. Moreover, in ASM and ABM, the levels were much higher in CSF than in serum for most of the mediators, even though the patients with ABM had positive blood cultures for the actual pathogen. Notably, in contrast to most of the mediators, IP-10 levels in CSF had the highest median value in the ASM group, indicating a potential role for this chemokine in aseptic meningitis. Finally, these changes in inflammatory mediators were associated with marked changes in KP metabolites in CSF. In particular, all groups of CNS infections had increased IDO activity assessed by KYN/TRP ratio compared with controls, indicating an increased catabolism of TRP. Interestingly, patients with encephalitis and viral meningitis had an unfavorable balance between neuroprotective and neurotoxic TRP metabolites. These changes in KP metabolites were associated with CSF levels of neopterin, and for the KYN/TRP ratio also with IP-10, suggesting a link between IFN- $\gamma$ and altered KP metabolites in these patients.

The influx of inflammatory cells and the resulting dysregulation of cytokine networks may be detrimental during CNS infection and is thought to contribute to neurological complications $[12,42-44]$. CNS inflammation is a complex process, depending on anatomical site (parenchyma vs meninges), cell type being involved (e.g., infiltrating leukocytes in acute infection vs brain-derived cells), and causing agent (e.g., viral vs bacterial). In this study, we describe findings from patients with acute inflammation in the CNS, mainly of infectious cause. The levels of cytokines in CSF were generally higher for all infectious groups compared to control, and strongly correlated with each other, demonstrating a general inflammatory response in patients with acute CNS infections. The pattern of increasing levels of inflammatory markers from encephalitis to aseptic meningitis and finally to bacterial meningitis indicates increased inflammation in all subgroup of patients, but with a more modest CSF inflammation in patients with encephalitis. Not surprisingly, the highest levels of most of the mediators were found in patients with bacterial meningitis and there was a CSF/serum ratio $>1$ of most mediators in patients with ABM, despite bacteremia. Furthermore, most cytokines were correlated with CSF WBC count, but not with corresponding serum levels, which implies the inflammatory response in the CNS to be independent of a peripheral immune response with no or minimal influx or efflux between the two compartments. These results indicate a predominately intrathecal production of these mediators in CNS infections. IL-6, IL-8, and TNF in CSF have in several studies been found to be elevated in meningitis and have even been proposed as biomarkers in CSF for bacterial meningitis [6, 45]. We found a general increase for most of the inflammatory mediators in both ASM and ABM, and our study does not support the use of one particular cytokine as a diagnostic marker to distinguish ABM from other CNS conditions, including ASM. 


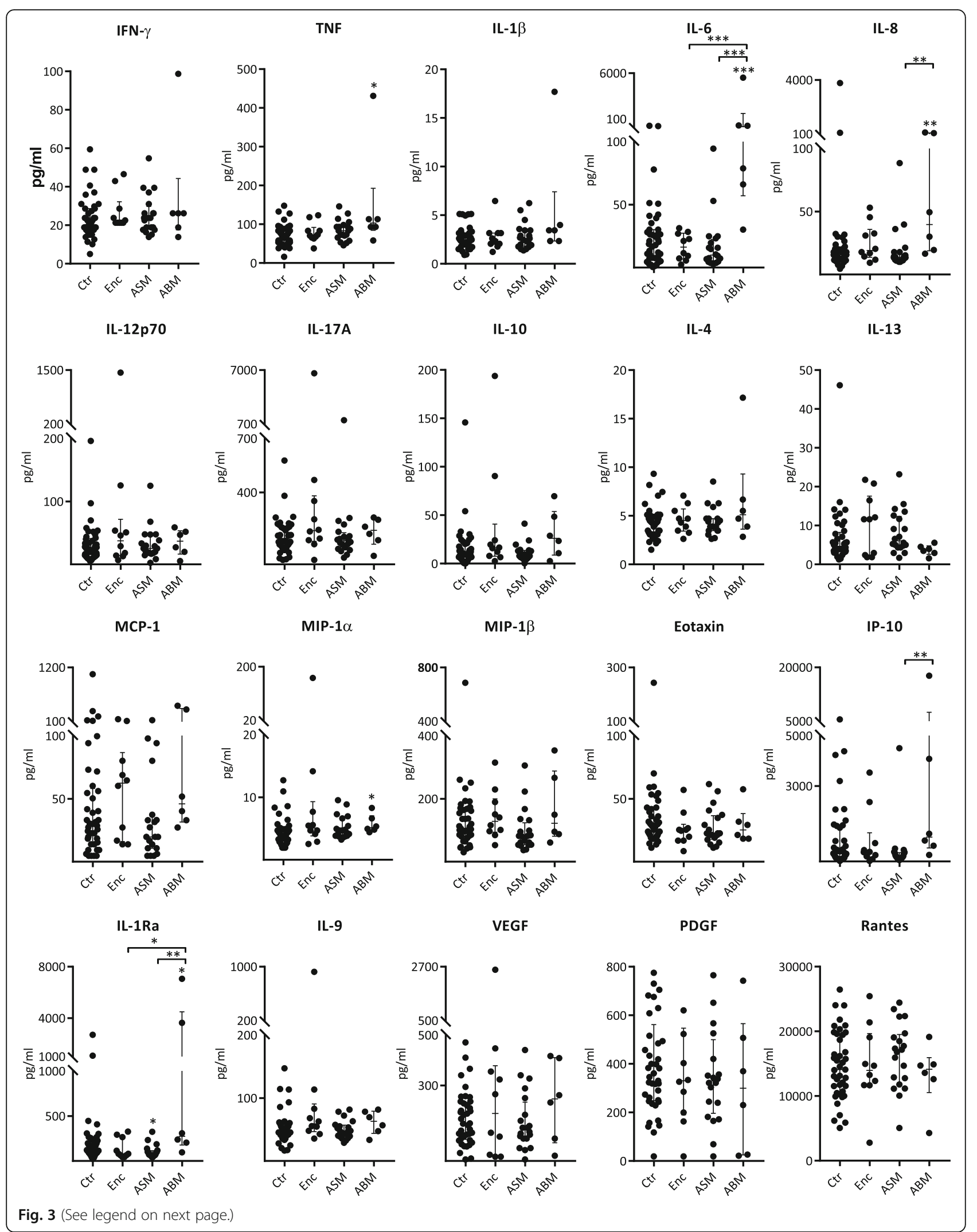


(See figure on previous page.)

Fig. 3 Cytokines in serum in patients with encephalitis (Enc, $n=10)$, aseptic meningitis (ASM, $n=20)$, bacterial meningitis (ABM, $n=6)$ in comparison with controls $(C t r, n=41)$. Data shown are medians with IQR. Asterisks above patient groups indicate significant difference vs controls, asterisks above horizontal lines indicate significant differences between individual groups (Mann-Whitney $U$ test): ${ }^{*} p<0.05,{ }^{* *} p<0.01$, and ${ }^{* * *} p<0.001$. Values below the detection limit were set to the lowest detectable level for that analyte

In contrast to most of the inflammatory markers, IP-10 levels were comparable in patients with ASM and ABM. IP-10 is secreted by several cell types in response to IFN- $\gamma$, and experimental studies have shown that IP-10 is highly induced in the CNS (e.g., West Nile infections [46], HIV encephalitis [47], HSV encephalitis [48], enteroviral encephalitis [3, 49], Japanese encephalitis [50], and tick-borne encephalitis [TBE] [51]). Increased levels have also been reported in patients suffering from TBE [52], neuroborreliosis [53], enterovirus infection [3], HSV meningitis, and HSV encephalitis [9]. IP-10 is a chemoattractant for monocytes and macrophages and promotes $\mathrm{T}$ cell recruitment, especially of $\mathrm{CD}^{+} \mathrm{T}$ cells $[46,54,55]$. Although IP-10 has been linked to viral clearance in the CNS [48, 55], IP-10 has also been described to promote apoptosis of neurons and excessive production has been associated with more severe outcome $[47,50,51]$. In the present study, we found an association between IP-10 and the KYN/TRP ratio, indicating increased IDO activity and catabolism of TRP with potential harmful effects on CNS. Thus, our present data may further support a potential role for this chemokine in CNS infections, particularly in ASM. This should be further investigated.

Neurologic dysfunction is common in patients with encephalitis and bacterial meningitis, and dysregulation of the KP has been shown in syndromes and disorders with certain overlap in symptoms [21, 30, 32, 56, 57]. Herein, we compared these metabolites in patients with a stringent definition of etiology; encephalitis, confirmed viral meningitis, and bacterial meningitis. Recent literature has shown that in HSV encephalitis, a low level of the neuroprotective metabolite KYNA was associated with more severe outcome [31]. In TB meningitis, low TRP was associated with a better survival rate [34]. In our study, the ratio of the neuroprotective metabolite KYNA to the sum of neurotoxic metabolites 3-HK and QA was lower for patients with encephalitis compared to the other groups, which could indicate a stronger activation of the KMO branch in encephalitis. In fact, studies are ongoing regarding the inhibition of several steps in the KP, including studies on centrally available KMO inhibitors [58].

In the present study, we observed very low levels of TRP for several patients with CNS infection, especially in patients with ASM. We hypothesize that this results from increased IDO activity, as these patients had a significantly higher level of KYN compared to patients with detectable TRP levels. The strong correlation of KYN/ TRP ratio with neopterin and IP-10 indicates that this IDO activity is driven by IFN- $\gamma$. IDO has been found to exhibit an immunosuppressive effect by upregulation of $\mathrm{T}_{\text {regs }}$ and downregulation of Th17 cells, which could be relevant in CNS infections, especially in ASM where T cells are of particular importance. In contrast to the association with IDO activity, neopterin, but not IP-10, was associated with a "neurotoxic" KYNA/(3-HK + QA) ratio in patients with $\mathrm{CNS}$ infection. The lack of correlation of IP-10 to $\mathrm{KYNA} /(3-\mathrm{HK}+\mathrm{QA})$ could be related to low statistical power. However, whereas IP-10 is induced by IFN- $\gamma$ in several cell types including monocytes, stromal cells, and endothelial cells [59], neopterin is almost selectively produced in monocytes/macrophages, and through its relation to tetrahydrobiopterin, neopterin may be more closely related to tryptophan metabolism than IP-10 [60, 61].

Finally, when looking at the CSF/serum ratios of KP metabolites, we found very narrow ranges in the control group, suggesting strict control of KP in healthy subjects.

Studies of the inflammatory profile in human CNS infections including both meningeal and parenchymal infections are relatively scarce [62-65], and comparisons between different studies are hampered by the diversity of causing agents and divergent inclusion criteria. Nevertheless, knowledge of immunological mechanism is pivotal in order to develop better diagnostic and potentially therapeutic tools for these patients. To our knowledge, the measurement of a large panel of metabolites in the $\mathrm{KP}$ in both serum and CSF, with parallel analyses of a wide range of cytokines and related mediators, have not been reported for patients with these conditions, especially not for encephalitis. Moreover, the correlation in present study of IP-10 and neopterin with the KP metabolites and the decreased $\mathrm{KYNA} /(3-\mathrm{HK}+\mathrm{QA})$ ratio in encephalitis are interesting findings that, as far as we are aware of, have not been reported in these clinical relevant CNS infections. However, the present study also has some limitations. The subgroups of patients, and in particular patients with ABM and encephalitis, were small and too small for sub-analyses on causing agents. Moreover, the etiology was unknown for $60 \%$ of patients with encephalitis with few patients with confirmed viral cause. Besides, due to lack of reliable measures of severity and outcome, together with the relatively low number of patients with CNS infection, we cannot make any 


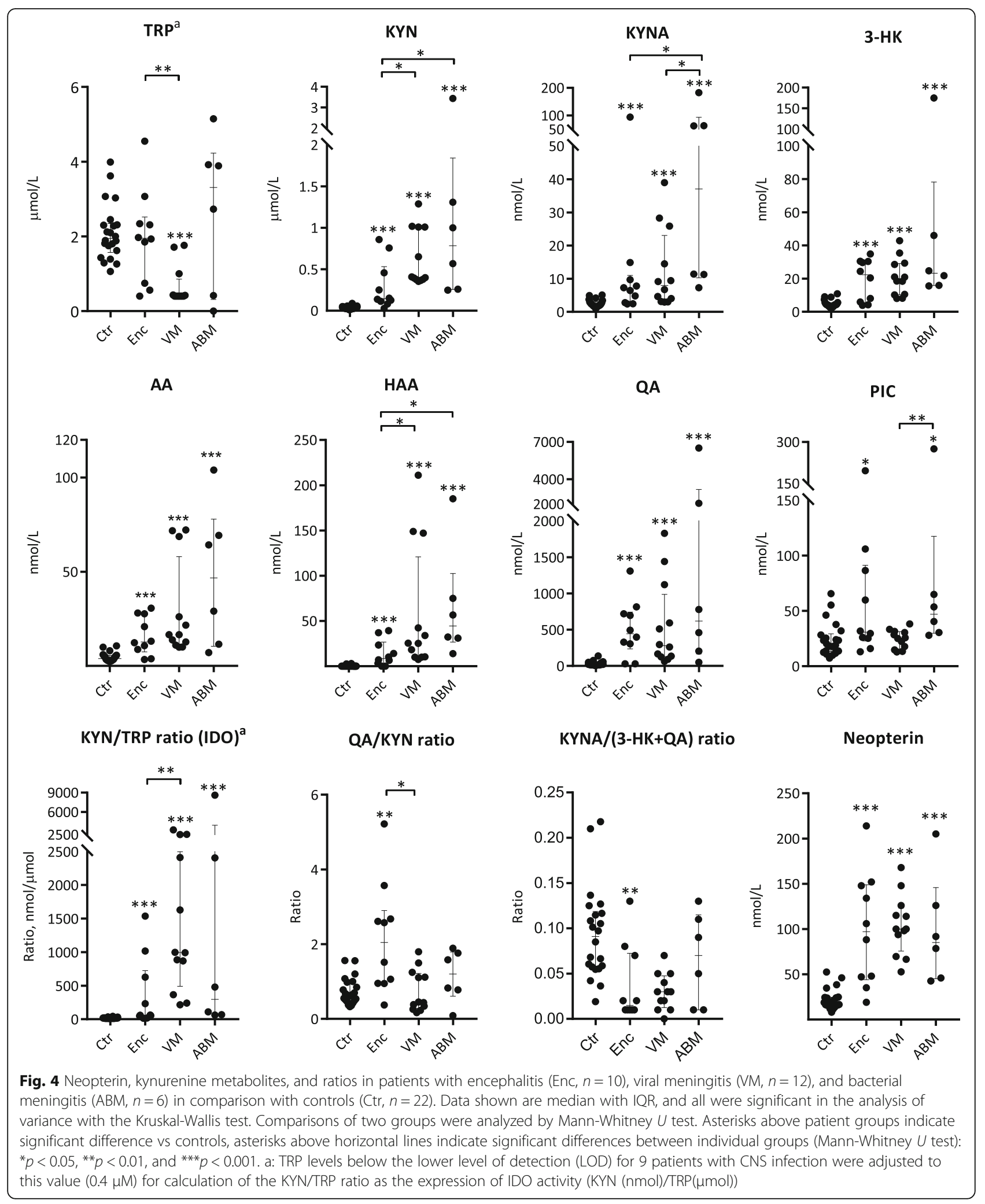

conclusion regarding the use of these cytokines and metabolites as prognostic markers. The control group in our study consisted of patients with similar presentation and no pleocytosis, but many of these patients suffered from systemic infections, as reflected by elevated serum neopterin levels. This may have camouflaged significant 
A Neopterin vs KYN/TRP-ratio (IDO)

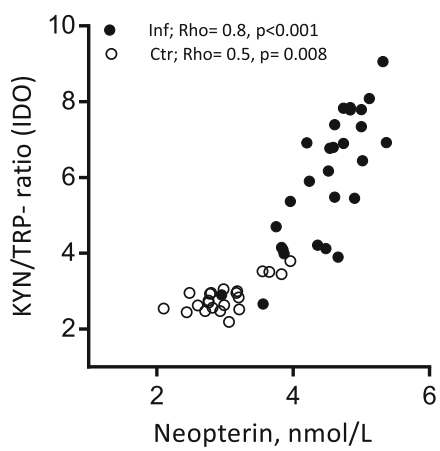

KYNA/(3HK+QA) vs Neopterin

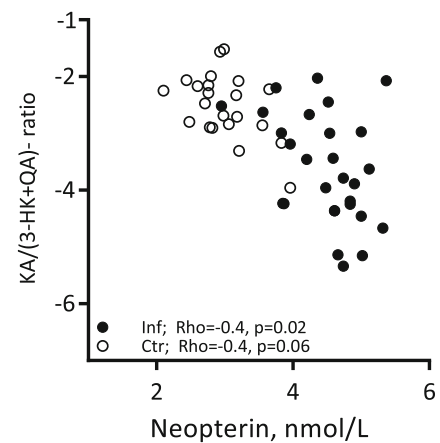

B

IP-10 vs KYN/TRP-ratio (IDO)

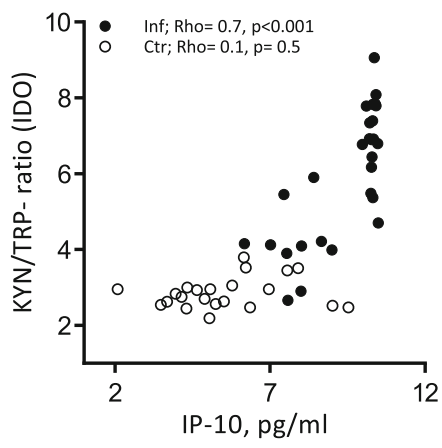

D $K Y N A /(3-H K+Q A)$ vs IP-10

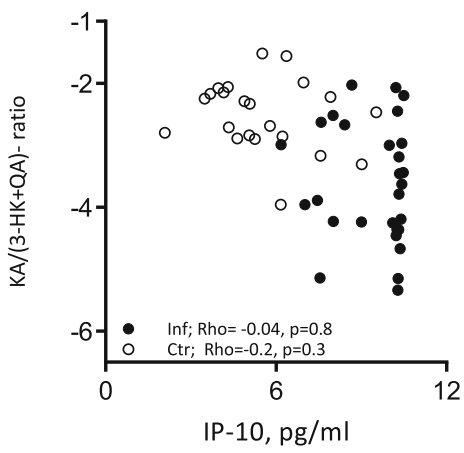

Fig. 5 Correlations of neopterin and IP-10, as markers of IFN-y activity, with activation of the KP. a Neopterin vs KYN/ TRP ratio (IDO) ( $n=50$; Rho 0.9, $p<0.001)$. b IP-10 vs KYN/TRP ratio (IDO) $(n=50 ;$ Rho $0.8, p<0.001)$. c Neopterin vs KYNA/(3-HK + QA) ratio $(n=50 ;$ Rho $-0.7, p<0.001)$. d IP-10 vs KYNA/(3-HK $+Q A)$ ratio $(n=50$, Rho $-0.5, p<0.001)$. Data shown are obtained by Spearman's rank correlation

findings in serum profiles. On the other hand, these controls may be more clinically relevant than healthy controls without any disease symptoms like fever and headache. Finally, correlations do not necessarily mean any causal relationship and more mechanistic studies as well as larger studies with samples also taken during follow-up are needed to make firmer conclusions.

\section{Conclusions}

In conclusion, we found a marked increase in a wide range of inflammatory mediators in CSF in aseptic and bacterial meningitis with a more modest increase in encephalitis. The markedly higher levels in CNS than in serum for most of the mediators indicate compartmentalization of immune responses in the CSF. Our data may also suggest that increased IFN- $\gamma$ activity, as assessed by neopterin and IP-10, may contribute to neurotoxicity through enhanced TRP catabolism. In particular, dysregulation of the KP with signs of an increased formation of neurotoxic QA in encephalitis should be explored further in these conditions.

\section{Additional files}

Additional file 1: Table S1. Case definitions of encephalitis, aseptic and viral meningitis and bacterial meningitis. (PDF $179 \mathrm{~kb}$ )

Additional file 2: Figure S1. Flowchart of inclusion of patients and overview of various analyses performed in the study population. (PDF 342 kb)

Additional file 3: Table S2. Cytokine levels in CSF and serum. (PDF $215 \mathrm{~kb}$ )

Additional file 4: Table S3. Correlations of cytokines in CSF with serum levels, CSF WBC, albumin ratio and KYN/TRP ratio. (PDF 197 kb)

Additional file 5: Table S4. KP metabolites in CSF and serum. (PDF $198 \mathrm{~kb}$ )

Additional file 6: Figure S2. Serum levels of KP metabolites. (PDF $81 \mathrm{~kb}$ )

Additional file 7: Table S5. CSF/serum ratios of KP metabolites and the correlation with CSF WBC and albumin ratio. (PDF 194 kb)

\section{Abbreviations}

3-HAA: 3-Hydroxyanthranilic acid; 3-HK: 3-Hydroxykynurenine; AA: Anthranilic acid; ABM: Acute bacterial meningitis; Albumin ratio: CSF albumin/serum albumin; ASM: Aseptic meningitis; Glucose ratio: CSF glucose/serum glucose; HAO: 3-Hydroxyanthranilic acid oxidase; HSV1: Herpes simplex 1 virus; IDO: Indoleamine 2,3-dioxygenase; KAT: Kynurenine aminotransferase; KMO: Kynurenine 3-monooxygenase; KP: Kynurenine pathway of tryptophan metabolism; KYN: Kynurenine; KYNA: Kynurenic acid; KYNU: Kynureninase; LDL: The lowest detectable level; LOD: Lower limit of detection; NMDAr: N- 
methyl-D-aspartate receptor; QA: Quinolinic acid; TRP: Tryptophan; VM: Viral meningitis; VZV: Varicella-zoster virus; WBC: White blood cell count; $X A$ : Xanthurenic acid

\section{Acknowledgements}

The Department of Infectious Diseases at Ullevaal Hospital represented by head of department professor Dag Kvale (MD, PhD) provided salary and office facilities to EQP during the study period. We would also like to thank laboratory staff at Bevital, Bergen Norway and the Research Laboratory, Nordland Hospital, Bodø, Norway for excellent service. The authors are especially grateful to nurses and physicians at the Emergency Room, the Medical Intensive Care Unit, the Department of Internal Medicine and the Department of Neurology at Oslo University Hospital, Ullevaal for their enthusiastic and friendly help in including patients during the study period. Finally, we thank the patients and next of kin who gave consent to participate in this study.

\section{Funding}

None.

\section{Availability of data and materials}

The dataset used during the current study is stored in a secured research data server at Oslo University Hospital. The datasets used are available from the corresponding author on reasonable request.

\section{Authors' contributions}

VO, EQP, PA, BS, OD, AMBK, and AMDR contributed to the study design. EQP, $V O$, and $O D$ contributed to the inclusion and evaluation of patients. TEM, $\varnothing \mathrm{M}$, and PMU contributed to the laboratory analyses. EQP, PA, TU, ØM, PMU, $T E M, B S$, and AMDR contributed to the data analysis and interpretation. EQP, $T U$, and TEM contributed to the statistical analyses. EQP, PA, and AMDR contributed to the writing of the manuscript. All authors contributed to the revision of the manuscript and approved the final version.

\section{Ethics approval and consent to participate}

The Regional Committees for Medical and Health Research Ethics (REC South East, reference number 2011/2578) and the ethical council of the hospital approved the study protocol. Written informed consent was obtained from all individual patients or their next of kin included in the study.

\section{Consent for publication}

Not applicable.

\section{Competing interests}

The authors declare that they have no competing interests.

\section{Publisher's Note}

Springer Nature remains neutral with regard to jurisdictional claims in published maps and institutional affiliations.
Author details
${ }^{1}$ Department of Infectious Diseases, Oslo University Hospital, Ullevaal Hospital, P. O. Box 4956 Nydalen, N-0450 Oslo, Norway. ${ }^{2}$ Institute of Clinical Medicine, University of Oslo, Oslo, Norway. ${ }^{3}$ Research Institute of Internal Medicine, Oslo University Hospital Rikshospitalet, Oslo, Norway. ${ }^{4}$ Section of Clinical Immunology and Infectious Diseases, Oslo University Hospital Rikshospitalet, Oslo, Norway. ${ }^{5}$ Faculty of Medicine, University of Oslo, Oslo, Norway. ${ }^{6}$ K.G. Jebsen Inflammatory Research Center, University of Oslo, Oslo, Norway. ${ }^{7}$ K.G. Jebsen Thrombosis Research and Expertise Center, Tromsø, Norway. ${ }^{8}$ Bevital A/S, Bergen, Norway. ${ }^{9}$ Department of Microbiology, Oslo University Hospital, Ullevaal, Oslo, Norway. ${ }^{10}$ Department of Acute Medicine, Oslo University Hospital, Ullevaal, Oslo, Norway. ${ }^{11}$ Department of Immunology, Oslo University Hospital, Oslo, Norway. ${ }^{12}$ Department of Clinical Science, University of Bergen, Bergen, Norway. ${ }^{13}$ Research Laboratory, Nordland Hospital, Bodø, Norway. ${ }^{14}$ Faculty of Health Sciences, University of Tromsø, Tromsø, Norway. ${ }^{15}$ Centre of Molecular Inflammation Research, Norwegian University of Science and Technology, Trondheim, Norway.

Received: 14 May 2018 Accepted: 11 November 2018 Published online: 23 November 2018

\section{References}

1. Asano T, Ichiki K, Koizumi S, Kaizu K, Hatori T, Fujino O, Mashiko K, Sakamoto $Y$, Miyasho T, Fukunaga Y. Enhanced expression of cytokines/chemokines in cerebrospinal fluids in mumps meningitis in children. Pediatr Int. 2011;53: $143-6$.

2. Asano T, Ichiki K, Koizumi S, Kaizu K, Hatori T, Fujino O, Mashiko K, Sakamoto Y, Miyasho T, Fukunaga Y. IL-17 is elevated in cerebrospinal fluids in bacterial meningitis in children. Cytokine. 2010;51:101-6.

3. Cavcic A, Tesovic G, Gorenec L, Grgic I, Benic B, Lepej SZ. Concentration gradient of CXCL10 and CXCL11 between the cerebrospinal fluid and plasma in children with enteroviral aseptic meningitis. Eur J Paediatr Neurol. 2011;15:502-7.

4. Coutinho LG, Christen S, Bellac CL, Fontes FL, Souza FR, Grandgirard D, Leib $\mathrm{SL}$, Agnez-Lima LF. The kynurenine pathway is involved in bacterial meningitis. J Neuroinflammation. 2014;11:169.

5. Ichiyama T, Maeba S, Suenaga N, Saito K, Matsubara T, Furukawa S. Analysis of cytokine levels in cerebrospinal fluid in mumps meningitis: comparison with echovirus type 30 meningitis. Cytokine. 2005;30:243-7.

6. Prasad R, Kapoor R, Srivastava R, Mishra OP, Singh TB. Cerebrospinal fluid TNF-alpha, IL-6, and IL-8 in children with bacterial meningitis. Pediatr Neurol. 2014;50:60-5.

7. Grandgirard D, Gaumann R, Coulibaly B, Dangy JP, Sie A, Junghanss T, Schudel H, Pluschke G, Leib SL. The causative pathogen determines the inflammatory profile in cerebrospinal fluid and outcome in patients with bacterial meningitis. Mediat Inflamm. 2013;2013:312476.

8. Shapiro S, Miller A, Lahat N, Sobel E, Lerner A. Expression of matrix metalloproteinases, sICAM-1 and IL-8 in CSF from children with meningitis. J Neurol Sci. 2003;206:43-8.

9. Lind L, Studahl M, Persson Berg L, Eriksson K. CXCL11 production in cerebrospinal fluid distinguishes herpes simplex meningitis from herpes simplex encephalitis. J Neuroinflammation. 2017;14:134.

10. Asaoka K, Shoji H, Nishizaka S, Ayabe M, Abe T, Ohori N, Ichiyama T, Eizuru $Y$. Non-herpetic acute limbic encephalitis: cerebrospinal fluid cytokines and magnetic resonance imaging findings. Intern Med. 2004;43:42-8.

11. Bociaga-Jasik M, Ciesla A, Kalinowska-Nowak A, Skwara P, Garlicki A, Mach T. Role of IL-6 and neopterin in the pathogenesis of herpetic encephalitis. Pharmacol Rep. 2011;63:1203-9.

12. Kamei S, Taira N, Ishihara M, Sekizawa T, Morita A, Miki K, Shiota H, Kanno A, Suzuki Y, Mizutani T, et al. Prognostic value of cerebrospinal fluid cytokine changes in herpes simplex virus encephalitis. Cytokine. 2009;46:187-93.

13. Michael BD, Griffiths MJ, Granerod J, Brown D, Davies NW, Borrow R, Solomon T. Characteristic cytokine and chemokine profiles in encephalitis of infectious, immune-mediated, and unknown aetiology. PLoS One. 2016;11: e0146288.

14. Schwarcz R, Bruno JP, Muchowski PJ, Wu HQ. Kynurenines in the mammalian brain: when physiology meets pathology. Nat Rev Neurosci. 2012:13:465-77.

15. Wang Q, Liu D, Song P, Zou MH. Tryptophan-kynurenine pathway is dysregulated in inflammation, and immune activation. Front Biosci (Landmark Ed). 2015;20:1116-43.

16. Frumento G, Rotondo R, Tonetti M, Damonte G, Benatti U, Ferrara GB. Tryptophan-derived catabolites are responsible for inhibition of $T$ and natural killer cell proliferation induced by indoleamine 2,3-dioxygenase. J Exp Med. 2002;196:459-68.

17. Terness P, Bauer TM, Rose L, Dufter C, Watzlik A, Simon H, Opelz G. Inhibition of allogeneic T cell proliferation by indoleamine 2,3-dioxygenaseexpressing dendritic cells: mediation of suppression by tryptophan metabolites. J Exp Med. 2002;196:447-57.

18. Fallarino F, Grohmann U, You S, McGrath BC, Cavener DR, Vacca C, Orabona C, Bianchi R, Belladonna ML, Volpi C, et al. The combined effects of tryptophan starvation and tryptophan catabolites down-regulate $T$ cell receptor zeta-chain and induce a regulatory phenotype in naive T cells. J Immunol. 2006;176:6752-61.

19. Pallotta MT, Orabona C, Volpi C, Vacca C, Belladonna ML, Bianchi R, Servillo G, Brunacci C, Calvitti M, Bicciato S, et al. Indoleamine 2,3-dioxygenase is a signaling protein in long-term tolerance by dendritic cells. Nat Immunol. $2011 ; 12: 870-8$. 
20. Guillemin GJ, Smythe G, Takikawa O, Brew BJ. Expression of indoleamine 2,3-dioxygenase and production of quinolinic acid by human microglia, astrocytes, and neurons. Glia. 2005;49:15-23.

21. Erhardt S, Blennow K, Nordin C, Skogh E, Lindstrom LH, Engberg G. Kynurenic acid levels are elevated in the cerebrospinal fluid of patients with schizophrenia. Neurosci Lett. 2001;313:96-8.

22. Linderholm KR, Skogh E, Olsson SK, Dahl ML, Holtze M, Engberg G, Samuelsson M, Erhardt S. Increased levels of kynurenine and kynurenic acid in the CSF of patients with schizophrenia. Schizophr Bull. 2012;38:426-32.

23. Savitz J, Dantzer R, Wurfel BE, Victor TA, Ford BN, Bodurka J, Bellgowan PS, Teague TK, Drevets WC. Neuroprotective kynurenine metabolite indices are abnormally reduced and positively associated with hippocampal and amygdalar volume in bipolar disorder. Psychoneuroendocrinology. 2015;52:200-11.

24. Olsson SK, Samuelsson M, Saetre P, Lindstrom L, Jonsson EG, Nordin C, Engberg G, Erhardt S, Landen M. Elevated levels of kynurenic acid in the cerebrospinal fluid of patients with bipolar disorder. J Psychiatry Neurosci. 2010;35:195-9.

25. Widner B, Leblhuber F, Fuchs D. Increased neopterin production and tryptophan degradation in advanced Parkinson's disease. J Neural Transm (Vienna). 2002;109:181-9.

26. Giil LM, Midttun $\mathrm{O}$, Refsum $H$, Ulvik A, Advani R, Smith AD, Ueland PM Kynurenine pathway metabolites in Alzheimer's disease. J Alzheimers Dis. 2017:60:495-504.

27. Guidetti P, Luthi-Carter RE, Augood SJ, Schwarcz R. Neostriatal and cortical quinolinate levels are increased in early grade Huntington's disease. Neurobiol Dis. 2004;17:455-61.

28. Heyes MP, Brew BJ, Martin A, Price RW, Salazar AM, Sidtis JJ, Yergey JA, Mouradian MM, Sadler AE, Keilp J, et al. Quinolinic acid in cerebrospinal fluid and serum in HIV-1 infection: relationship to clinical and neurological status. Ann Neurol. 1991;29:202-9.

29. Valle M, Price RW, Nilsson A, Heyes M, Verotta D. CSF quinolinic acid levels are determined by local HIV infection: cross-sectional analysis and modelling of dynamics following antiretroviral therapy. Brain. 2004;127: 1047-60.

30. Yan EB, Frugier T, Lim CK, Heng B, Sundaram G, Tan M, Rosenfeld JV, Walker DW, Guillemin GJ, Morganti-Kossmann MC. Activation of the kynurenine pathway and increased production of the excitotoxin quinolinic acid following traumatic brain injury in humans. J Neuroinflammation. 2015;12:110.

31. Atlas A, Franzen-Rohl E, Soderlund J, Jonsson EG, Samuelsson M, Schwieler L, Skoldenberg B, Engberg G. Sustained elevation of kynurenic acid in the cerebrospinal fluid of patients with herpes simplex virus type 1 encephalitis. Int J Tryptophan Res. 2013;6:89-96.

32. Halperin JJ, Heyes MP. Neuroactive kynurenines in Lyme borreliosis. Neurology. 1992;42:43-50.

33. Holtze M, Mickiene A, Atlas A, Lindquist L, Schwieler L. Elevated cerebrospinal fluid kynurenic acid levels in patients with tick-borne encephalitis. J Intern Med. 2012;272:394-401.

34. van Laarhoven A, Dian S, Aguirre-Gamboa R, Avila-Pacheco J, Ricano-Ponce I, Ruesen C, Annisa J, Koeken V, Chaidir L, Li Y, et al. Cerebral tryptophan metabolism and outcome of tuberculous meningitis: an observational cohort study. Lancet Infect Dis. 2018;18:526-35.

35. Midttun O, Hustad S, Ueland PM. Quantitative profiling of biomarkers related to B-vitamin status, tryptophan metabolism and inflammation in human plasma by liquid chromatography/tandem mass spectrometry. Rapid Commun Mass Spectrom. 2009;23:1371-9.

36. Bevital AS [http://www.bevital.no]. Accessed 19 Nov 2018.

37. Yamada A, Akimoto H, Kagawa S, Guillemin GJ, Takikawa O. Proinflammatory cytokine interferon-gamma increases induction of indoleamine 2,3-dioxygenase in monocytic cells primed with amyloid beta peptide 1-42: implications for the pathogenesis of Alzheimer's disease. J Neurochem. 2009;110:791-800.

38. Guillemin GJ, Kerr SJ, Pemberton LA, Smith DG, Smythe GA, Armati PJ, Brew BJ. IFN-beta1b induces kynurenine pathway metabolism in human macrophages: potential implications for multiple sclerosis treatment. J Interf Cytokine Res. 2001;21:1097-101.

39. Fuchs D, Weiss G, Wachter H. Neopterin, biochemistry and clinical use as a marker for cellular immune reactions. Int Arch Allergy Immunol. 1993;101:1-6.

40. Raison CL, Dantzer R, Kelley KW, Lawson MA, Woolwine BJ, Vogt G, Spivey JR, Saito K, Miller AH. CSF concentrations of brain tryptophan and kynurenines during immune stimulation with IFN-alpha: relationship to CNS immune responses and depression. Mol Psychiatry. 2010;15:393-403.
41. Capuron L, Schroecksnadel S, Feart C, Aubert A, Higueret D, BarbergerGateau P, Laye S, Fuchs D. Chronic low-grade inflammation in elderly persons is associated with altered tryptophan and tyrosine metabolism: role in neuropsychiatric symptoms. Biol Psychiatry. 2011;70:175-82.

42. Fitch MT, Silver J. CNS injury, glial scars, and inflammation: inhibitory extracellular matrices and regeneration failure. Exp Neurol. 2008;209:294-301.

43. Skoldenberg B, Aurelius E, Hjalmarsson A, Sabri F, Forsgren M, Andersson B, Linde A, Strannegard O, Studahl M, Hagberg L, Rosengren L. Incidence and pathogenesis of clinical relapse after herpes simplex encephalitis in adults. J Neurol. 2006;253:163-70.

44. Koedel U, Scheld WM, Pfister HW. Pathogenesis and pathophysiology of pneumococcal meningitis. Lancet Infect Dis. 2002;2:721-36.

45. Pinto Junior VL, Rebelo MC, Gomes RN, Assis EF, Castro-Faria-Neto HC, Boia MN. IL-6 and IL-8 in cerebrospinal fluid from patients with aseptic meningitis and bacterial meningitis: their potential role as a marker for differential diagnosis. Braz J Infect Dis. 2011;15:156-8

46. Klein RS, Lin E, Zhang B, Luster AD, Tollett J, Samuel MA, Engle M, Diamond MS. Neuronal CXCL10 directs CD8+ T-cell recruitment and control of West Nile virus encephalitis. J Virol. 2005;79:11457-66.

47. Sui Y, Potula R, Dhillon N, Pinson D, Li S, Nath A, Anderson C, Turchan J, Kolson D, Narayan O, Buch S. Neuronal apoptosis is mediated by CXCL10 overexpression in simian human immunodeficiency virus encephalitis. Am J Pathol. 2004;164:1557-66.

48. Lokensgard JR, Hu S, Sheng W, vanOijen M, Cox D, Cheeran MC, Peterson PK. Robust expression of TNF-alpha, IL-1beta, RANTES, and IP-10 by human microglial cells during nonproductive infection with herpes simplex virus. J Neuro-Oncol. 2001;7:208-19.

49. Kothur K, Wienholt L, Mohammad SS, Tantsis EM, Pillai S, Britton PN, Jones CA, Angiti RR, Barnes EH, Schlub T, et al. Utility of CSF cytokine/chemokines as markers of active intrathecal inflammation: comparison of demyelinating, anti-NMDAR and enteroviral encephalitis. PLoS One. 2016;11:e0161656.

50. Bhowmick S, Duseja R, Das S, Appaiahgiri MB, Vrati S, Basu A. Induction of IP-10 (CXCL10) in astrocytes following Japanese encephalitis. Neurosci Lett. 2007:414:45-50.

51. Palus M, Vojtiskova J, Salat J, Kopecky J, Grubhoffer L, Lipoldova M, Demant $P$, Ruzek D. Mice with different susceptibility to tick-borne encephalitis virus infection show selective neutralizing antibody response and inflammatory reaction in the central nervous system. J Neuroinflammation. 2013;10:77.

52. Lepej SZ, Misic-Majerus L, Jeren T, Rode OD, Remenar A, Sporec V, Vince A. Chemokines CXCL10 and CXCL11 in the cerebrospinal fluid of patients with tick-borne encephalitis. Acta Neurol Scand. 2007;115:109-14.

53. Lepej SZ, Rode OD, Jeren T, Vince A, Remenar A, Barsic B. Increased expression of CXCR3 and CCR5 on memory CD4+ T-cells migrating into the cerebrospinal fluid of patients with neuroborreliosis: the role of CXCL10 and CXCL11. J Neuroimmunol. 2005;163:128-34.

54. Zhang B, Chan YK, Lu B, Diamond MS, Klein RS. CXCR3 mediates regionspecific antiviral T cell trafficking within the central nervous system during West Nile virus encephalitis. J Immunol. 2008;180:2641-9.

55. Dufour JH, Dziejman M, Liu MT, Leung JH, Lane TE, Luster AD. IFN-gammainducible protein 10 (IP-10; CXCL10)-deficient mice reveal a role for IP-10 in effector T cell generation and trafficking. J Immunol. 2002;168:3195-204.

56. Cuartero MI, de la Parra J, Garcia-Culebras A, Ballesteros I, Lizasoain I, Moro MA. The kynurenine pathway in the acute and chronic phases of cerebral ischemia. Curr Pharm Des. 2016;22:1060-73.

57. Holmberg D, Franzen-Rohl E, Idro R, Opoka RO, Bangirana P, Sellgren CM, Wickstrom R, Farnert A, Schwieler L, Engberg G, John CC. Cerebrospinal fluid kynurenine and kynurenic acid concentrations are associated with coma duration and long-term neurocognitive impairment in Ugandan children with cerebral malaria. Malar J. 2017;16:303.

58. Vecsei L, Szalardy L, Fulop F, Toldi J. Kynurenines in the CNS: recent advances and new questions. Nat Rev Drug Discov. 2013;12:64-82.

59. Liu M, Guo S, Hibbert JM, Jain V, Singh N, Wilson NO, Stiles JK. CXCL10/IP-10 in infectious diseases pathogenesis and potential therapeutic implications. Cytokine Growth Factor Rev. 2011;22:121-30.

60. Vancassel S, Capuron L, Castanon N. Brain kynurenine and BH4 pathways: relevance to the pathophysiology and treatment of inflammation-driven depressive symptoms. Front Neurosci. 2018;12:499.

61. Weiss G, Murr C, Zoller H, Haun M, Widner B, Ludescher C, Fuchs D. Modulation of neopterin formation and tryptophan degradation by Th1and Th2-derived cytokines in human monocytic cells. Clin Exp Immunol. 1999; 116:435-40. 
62. Kothur K, Wienholt L, Brilot F, Dale RC. CSF cytokines/chemokines as biomarkers in neuroinflammatory CNS disorders: a systematic review. Cytokine. 2015;77:227-37.

63. Azumagawa K, Suzuki S, Tanabe T, Wakamiya E, Kawamura N, Tamai H Neopterin, biopterin, and nitric oxide concentrations in the cerebrospinal fluid of children with central nervous system infections. Brain and Development. 2003;25:200-2.

64. Ostergaard C, Benfield T. Macrophage migration inhibitory factor in cerebrospinal fluid from patients with central nervous system infection. Crit Care. 2009;13:R101.

65. Matsuzono Y, Narita M, Akutsu Y, Togashi T. Interleukin-6 in cerebrospinal fluid of patients with central nervous system infections. Acta Paediatr. 1995; 84:879-83.

Ready to submit your research? Choose BMC and benefit from:

- fast, convenient online submission

- thorough peer review by experienced researchers in your field

- rapid publication on acceptance

- support for research data, including large and complex data types

- gold Open Access which fosters wider collaboration and increased citations

- maximum visibility for your research: over $100 \mathrm{M}$ website views per year

At $\mathrm{BMC}$, research is always in progress.

Learn more biomedcentral.com/submissions 